\section{Giant lymph node hyperplasia of the lung (Castleman's disease) associated with recurrent pleural effusion}

\author{
A A Awotedu, B A Otulana, C O Ukoli
}

\begin{abstract}
A case of giant lymph node hyperplasia (Castleman's disease) of the lung presented with pleural effusion (which was recurrent), an unusual complication. The patient was treated with pneumonectomy and has survived for three years without relapse. This is the first report of the disease from black Africa.
\end{abstract}

Since the original description in 1956 of the rare giant lymph node hyperplasia known as Castleman's disease, ${ }^{1}$ cases have been reported from various parts of the world. ${ }^{2-4}$ Although some of the patients were of negroid origin, no previous patients, to the best of our knowledge, have come from black Africa.

Medicine, University

College Hospital,

Ibadan, Nigeria

A A Awotedu

B A Otulana

C O Ukoli

Address for reprint requests: Dr B A Otulana, Respiratory Physiology Despiratory

Papworth Hospital

Pamborth Hospital,

Cambridge CB3 8RE.
Accepted 27 April 1990

\section{Case report}

A 21 year old black man who had been permanently resident in Africa was seen with a two week history of left sided pleuritic chest pain and fever. There was no cough, haemoptysis, or dyspnoea. Weight loss occurred only transiently at the onset of the

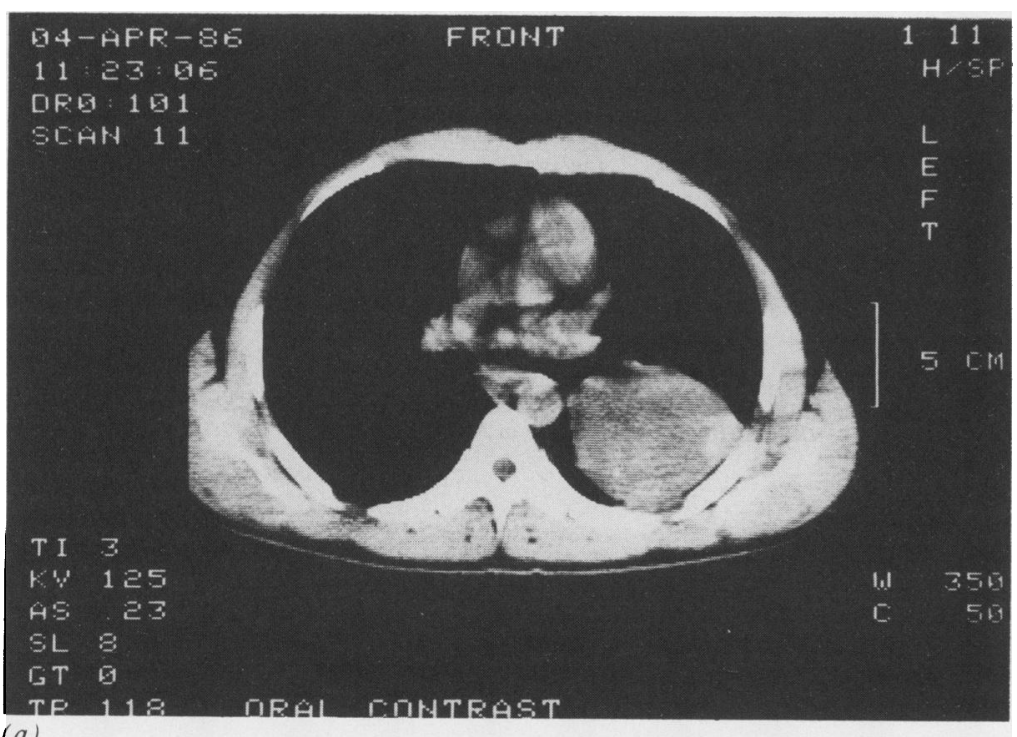

illness. The patient had no history of contact with tuberculosis or exposure to asbestos or other industrial agents. He was a non-smoker. On examination he was thin but otherwise looked well. He had no palpable lymph nodes, but did have signs of a left sided pleural effusion.

The chest radiograph (fig $1 b$ ) confirmed the left pleural effusion and showed a left perihilar mass with a convex border distinct from the pleural fluid. Computed tomography of the thorax showed a mass in the apical segment of the left lower lobe, some small hilar lymph nodes, and a left sided pleural effusion (fig 1a). Fibreoptic bronchoscopy showed nothing abnormal. The packed cell volume was 0.45 , erythrocyte sedimentation rate $85 \mathrm{~mm}$ in one hour, and white cell count 4.9 $\times 10^{9} / 1$ with a normal differential cell count. His Mantoux test (1 in 10000$)$ gave an $8 \mathrm{~mm}$ response.

The pleural fluid was straw coloured with a protein concentration of $66 \mathrm{~g} / \mathrm{l}$; no organisms were found by Gram or Ziehl-Neelsen staining or on cultures. Cytological examination showed numerous lymphocytes, histiocytes, and lymphoblasts. Pleural biopsy showed chronic inflammatory changes but no caseous granulomas or malignant cells.

Aspiration of the pleural fluid on two occasions (total yield 3.2 litres) was followed by reaccumulation over a few weeks. A four week therapeutic trial of antituberculous drugs did not alter the clinical picture so it was discontinued.

At thoracotomy a large, soft, spongy mass was found in the left lobe with enlarged hilar nodes; both were biopsied. Microscopically the lung mass and the lymph nodes showed angiofollicular hyperplasia, the follicles being composed mainly of small lymphocytes but

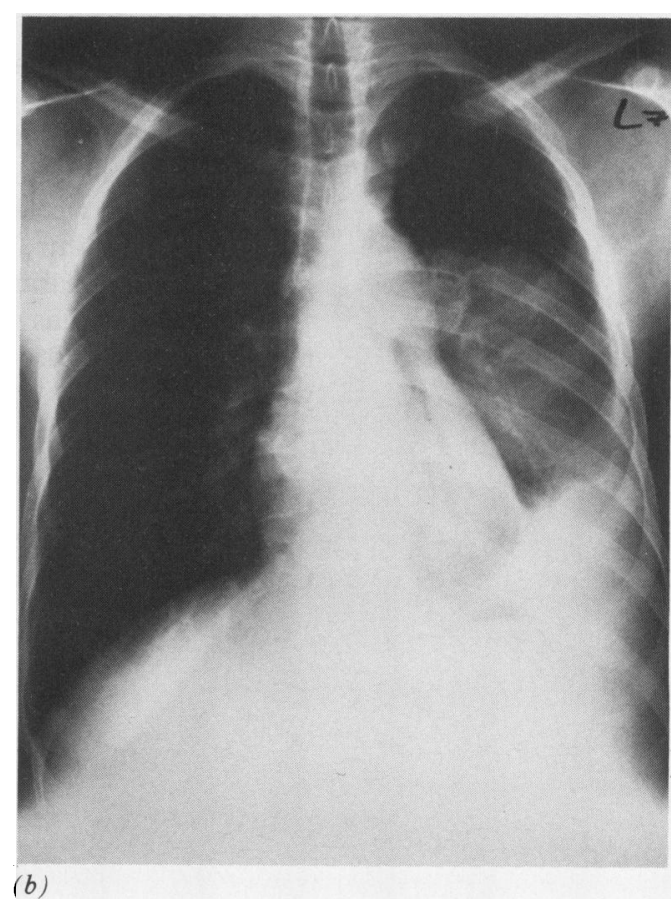

Figure 1 (a) Computed tomogram showing the mass in the apical segment of the left lower lobe. (b) Posteroanterior view chest radiograph showing the mass lesion with convex border in the left lung. 


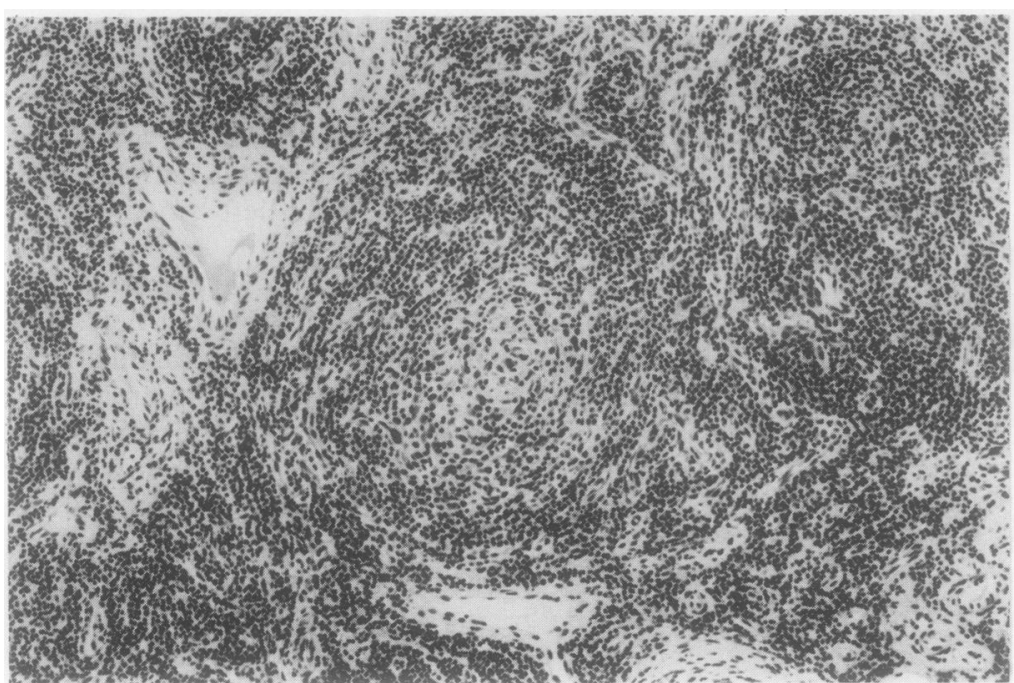

(a)

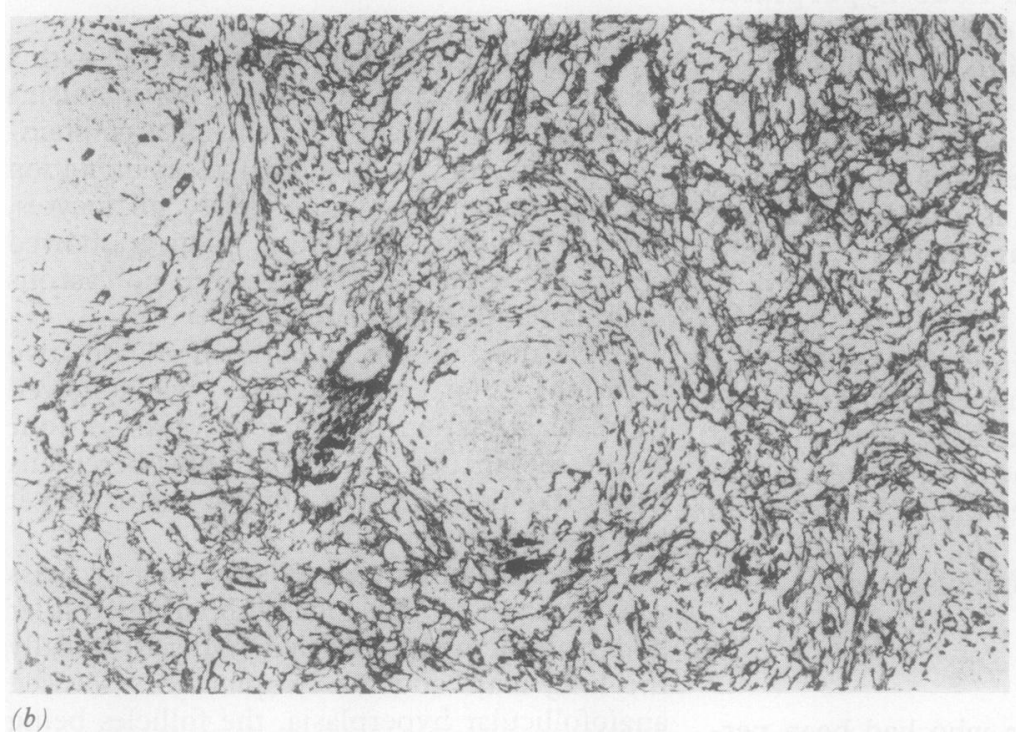

Figure 2 (a) Photomicrograph showing pseudofollicle with concentric rings of small lymphocytes. (Haematoxylin and eosin.) (b) Photomicrograph showing the follicular architecture and some interfollicular blood vessels. (Reticulin stain.)

some containing hyalinised vessels (fig 2). A histological diagnosis of benign giant lymph node hyperplasia of the lung (Castleman's disease) was made. Left pneumonectomy was carried out two weeks later in view of the large size of the tumour, which showed the same pathological appearances.

The patient has remained well with no recurrence of symptoms or signs for three years.

\section{Discussion}

The classic localised disease described by Castleman $^{1}$ has a mainly hyaline-vascular histological pattern and has been classified as type 2 . The plasma cell type, consisting predominantly of polyclonal plasma cells, has been recognised more recently..$^{56}$

Type 2 Castleman's disease accounts for about $90 \%$ of cases and tends to be benign, and surgical excision is in most instances curative. Type 1 is usually multicentric, with systemic and other manifestations such as anaemia, hypergammaglobulinaemia, amyloidosis, and jaundice.

The thorax is the most common site for the disease to occur, ${ }^{7}$ accounting for $60 \%$ of cases, but the tumour has been encountered in the retroperitoneal space, mesentery, skeletal muscles, and pelvis.

Our patient had a short illness. The clinical and laboratory findings were similar to those reported in most cases of type 2 Castleman's disease $^{235}$ except for the recurrent pleural effusion, which is a rare manifestation of the disease. It was found in only two of the 81 cases described by Keller et al. ${ }^{5}$

In black Africa tuberculosis is the most common cause of an exudative pleural effusion $^{8}$ and patients are often started empirically on antituberculous drugs after clinical exclusion of less common causes. Laboratory investigations and a therapeutic trial of antituberculous drugs did not support tuberculosis as the cause of the pleural effusion in our patient. The prompt response to pneumonectomy and the absence of relapse during the three year follow up also argue against tuberculosis as an additional cause of the lung disease in this case.

We thank Drs John Costello and John Keates for assistance in the management of the patient.

1 Castleman B, Iverson L, Menendez VP. Localised mediastinal lymph node hyperplasia resembling thymoma. Cancer 1956;9:822-30.

2 Gaba AR, Stein RS, Sweet DL, Variakojis D. Multicentric giant lymph node hyperplasia. Am J Clin Pathol 1978;69: 86-90.

3 Weisenburger DD, Nathwani BN, Winberg CD, Rapport H. Multicentric angiofollicular lymph node hyperplasia: a clinicopathologic study of 16 cases. Hum Pathol 1985; 16:162-72.

4 Friedman L, Dubowitz B, Papert B, Porteous P. Computerized tomography of Castleman's disease simulating a false renal artery aneurysm: a case report. J Urol 1987; 138:123-4.

5 Keller AR, Hochholzer L, Castleman B. Hyaline-vascular and plasma cell types of giant lymph node hyperplasia of the mediastinum and other locations. Cancer 1972;29: 670-83.

6 Frizzera G. Castleman's disease: more questions than answers. Hum Pathol 1985;16:202-5.

7 Anagnostou D, Harrison CV. Angiofollicular lymph node hyperplasia (Castleman). J Clin Pathol 1972;25:306-11.

8 Onadeko BO. A preliminary study of the pattern of pleural effusion in Africans. Nig Med J 1977;7:138-40. 